\title{
Differences in navigation performance and postpartal striatal volume associated with pregnancy in humans
}

\author{
Nina Lisofsky ${ }^{\mathrm{a}, \mathrm{c}, *}$, Jan Wiener ${ }^{\mathrm{b}}$, Olivier de Condappa ${ }^{\mathrm{b}}$, Jürgen Gallinat ${ }^{\mathrm{c}}$, Ulman Lindenberger ${ }^{\mathrm{a}, \mathrm{d}}$, \\ Simone Kühn ${ }^{\mathrm{a}, \mathrm{c}}$ \\ ${ }^{a}$ Max Planck Institute for Human Development, Berlin, Germany \\ ${ }^{\mathrm{b}}$ Department of Psychology, Bournemouth University, United Kingdom \\ ${ }^{\mathrm{c}}$ Department of Psychiatry and Psychotherapy, University Medical Center Hamburg-Eppendorf, Germany \\ ${ }^{\mathrm{d}}$ European University Institute, San Domenico di Fiesole, Italy
}

\section{A R T I C L E I N F O}

\section{Article history:}

Received 4 January 2016

Revised 8 August 2016

Accepted 28 August 2016

Available online 7 September 2016

\section{Keywords:}

Pregnancy

Striatum

Estrogen

Spatial learning strategies

SMRI

\begin{abstract}
A B S T R A C T
Pregnancy is accompanied by prolonged exposure to high estrogen levels. Animal studies have shown that estrogen influences navigation strategies and, hence, affects navigation performance. High estrogen levels are related to increased use of hippocampal-based allocentric strategies and decreased use of striatal-based egocentric strategies. In humans, associations between hormonal shifts and navigation strategies are less well studied. This study compared 30 peripartal women (mean age 28 years) to an age-matched control group on allocentric versus egocentric navigation performance (measured in the last month of pregnancy) and gray matter volume (measured within two months after delivery). None of the women had a previous pregnancy before study participation. Relative to controls, pregnant women performed less well in the egocentric condition of the navigation task, but not the allocentric condition. A whole-brain group comparison revealed smaller left striatal volume (putamen) in the peripartal women. Across the two groups, left striatal volume was associated with superior egocentric over allocentric performance. Limited by the cross-sectional study design, the findings are a first indication that human pregnancy might be accompanied by structural brain changes in navigation-related neural systems and concomitant changes in navigation strategy.
\end{abstract}

(c) 2016 Elsevier Inc. All rights reserved.

\section{Introduction}

Many studies have shown that variations in gonadal hormones in female animals -in particular estrogen- lead to structural brain changes, and to changes in behavior (Darnaudéry et al., 2007; DeVoogd \& Nottebohm, 1981; Fader, Hendricson, \& Dohanich, 1998; Galea \& Kavaliers, 1995; Galea et al., 2000; Gibbs, 2000; Korol, 2004; Luine \& Frankfurt, 2012; Pawluski, Brummelte, Barha, Crozier, \& Galea, 2009; Woolley, 1998; Woolley \& McEwen, 1993). Endogenous estrogen fluctuates naturally during the estrous cycle, peaks during pregnancy, and declines at menopause. Effects of varying estrogen levels therefore influence virtually all females during different phases of the lifespan. The hippocampus is one of the main targets of estrogen action in the brain, and its structure and function is affected by estrogen

* Corresponding author at: Medical Center Hamburg-Eppendorf, Martinistraße 52, 20246 Hamburg, Germany.

E-mail address: N.Lisofsky@uke.de (N. Lisofsky). variation (McEwen, 2002; Pawluski et al., 2009). Together with the striatum, the hippocampus plays a crucial role in spatial learning and navigation (Keefe, Burgess, Donnett, Jeffery, \& Maguire, 1998; McDonald \& White, 1994). The striatum also contains estrogen receptors, and it's function is modulated by estrogen (Küppers \& Beyer, 1999; Van Hartesveldt \& Joyce, 1986). It appears that estrogen also regulates the relative contributions of striatal and hippocampal structures to spatial learning, at least in rodents (e.g., Daniel \& Lee, 2004).

To understand how estrogen affects navigation, it is important to disentangle hippocampal and striatal contributions to spatial learning. While learning to navigate in a maze, navigators can associate motor responses with particular environmental cues and later react in response to these cues, resulting in an egocentric strategy, also called response learning (Kesner, Farnsworth, \& DiMattia, 1989; Restle, 1957). Alternatively, navigators can make use of associations between landmarks and places to build a cognitive map. This process is referred to as an allocentric strategy, or place learning (Kesner et al., 1989). Whereas the striatum is involved in stimulus-response learning and egocentric strategy use (Packard, 
2009), the hippocampus contains place cells required for allocentric navigation (Keefe et al., 1998). Korol and colleagues showed that estrogen differentially influences place and response learning in rats by shifting the relative contributions of the hippocampus and striatum during learning (Korol, 2004; Korol \& Kolo, 2002). Further studies replicated and extended this finding, revealing that high estrogen levels foster hippocampusdependent (place) strategies and decrease performance in striatum-dependent tasks, whereas low estrogen levels facilitate the use of striatum-dependent strategies (Daniel \& Lee, 2004; Galea et al., 2001; Zurkovsky, Brown, Boyd, Fell, \& Korol, 2007; for a review, see Hussain, Shams, \& Brake, 2014). Given that the largest shifts in endogenous estrogen levels in humans appear during pregnancy we set out to explore navigation performance shortly before delivery and navigation-related brain structures shortly after delivery.

Despite the strong body of literature on estrogen affecting navigation strategies in animals, almost nothing is known about the estrogen-navigation association in humans. The dissociation between hippocampus-based allocentric strategies and striatumbased egocentric strategies also holds in human navigation (Iaria, Petrides, Dagher, Pike, \& Bohbot, 2003; Wolbers \& Hegarty, 2010). Estrogen effects on the human brain have only begun to be studied and our understanding is far from being comprehensive. Initial structural magnetic resonance imaging (MRI) studies have found hippocampal structural plasticity between high-estrogen and low-estrogen phases of the menstrual cycle (Lisofsky et al., 2015; Protopopescu et al., 2008).

To our knowledge, no study thus far has investigated how variations in estrogen relate to navigation strategies in humans. This might be due to the difficulty to manipulate or observe human estrogen changes that are large enough to potentially affect brain and behavior to a measurable extent. Pregnancy is one instance of naturally occurring high estrogen level change. This event bears the potential to reveal whether hormonal shifts in human females are associated with alterations in the neural substrates and learning strategies underlying navigation performance. No study so far has investigated navigation strategy or performance differences in relation to human pregnancy, but pregnancy-related differences in other cognitive abilities, such as memory, have been addressed (e.g., Henry \& Rendell, 2007).

The present study had two main goals: First, to explore navigation strategies in pregnant women; second, to relate these strategies to alterations in brain structure observed shortly after delivery. We expected differences in route learning performance in pregnant relative to non-pregnant women. Specifically, we hypothesized a shift towards allocentric strategies during pregnancy, that is, lower performance in navigation tasks that are known to recruit egocentric strategies and better performance in tasks that require the use of allocentric strategies relative to the control group. We also expected pregnancy-related volumetric alterations in hippocampal and potentially striatal volume. Here, we expected that the hypothesized shift towards allocentric strategies during pregnancy would be associated with increased hippocampal volume, decreased striatal volume, or both.

Besides the two main study goals, we wanted to investigate potential differences in other cognitive abilities between pregnant and non-pregnant women. Since the previous literature on cognitive performance of pregnant women is contradictory (Christensen, Leach, \& Mackinnon, 2010; Glynn, 2010; Henry \& Rendell, 2007; Logan, Hill, Jones, Holt-Lunstad, \& Larson, 2014) and it is not clear yet, whether pregnancy goes along with reliable performance decreases in specific cognitive abilities. Therefore, we included an extensive cognitive test battery to compare performance of pregnant and non-pregnant women in an exploratory manner.

\section{Materials and methods}

\subsection{Participants}

Thirty healthy pregnant women participated in the study (age: 28.03 ( \pm 3.33$)$, mean years of education: $18.58( \pm 2.79)$ ). None of the women in the peripartal group have been pregnant before (>8 weeks of pregnancy). A group of thirty naturally cycling women that have also never been pregnant before was recruited as the control group (age: $27.97( \pm 3.37)$, years of education: $19.35( \pm 2.71)$ ). None of the women in the control group used hormonal contraceptives during the six month prior to study participation and within the study phase. None of the participants had a history of neurological or psychiatric conditions or drug/alcohol abuse. The study was conducted according to the Helsinki Declaration of 1975, as revised 2008, with approval from the Ethics Committee of the German Society for Psychology.

\subsection{Design and procedure}

Participants were tested with a number of cognitive tasks and questionnaires. The women in the peripartal group were tested during the last month of pregnancy. The assessment was scheduled based on the expected date of delivery. The MRI assessment was delayed to the first two months following delivery. The cognitive session lasted about 2.5-3.5 h, depending on individual pace of the participant. During the cognitive and the MRI session, women provided three saliva samples. For the control group, the imaging and cognitive measurements were scheduled on one or two days within the early follicular phase of their menstrual cycle (1-10 days after onset of menses). The procedures during the cognitive and imaging session were the same for both groups.

\subsection{Hormonal assessment}

Saliva samples were collected using SaliCaps collection devices (IBL-International, Hamburg, Germany), which are validated for sampling of steroid hormones. To account for the pulsative secretion of estrogen, we collected three samples spread over the $2 \mathrm{~h}$ testing time and pooled them afterwards, to minimize the effect of short-term fluctuations of hormone concentrations. Immediately after collection, saliva samples were frozen and stored at $-25^{\circ} \mathrm{C}$. The estrogen concentrations were determined by a commercial company (IBL-International, using IBL Saliva Immunoassay $-17 \beta$-Estradiol Saliva ELISA kit). Saliva samples were analyzed for all women in the peripartal group $(\mathrm{N}=30)$ and a subgroup of women in the control group $(\mathrm{N}=14)$. A two-sample $t$-test was used to compare estrogen levels between the two groups.

\subsection{Cognitive tasks and questionnaire}

We used a number of memory tasks, assessing episodic verbal memory (word-list recall (Schmiedek, Lövdén, \& Lindenberger, 2010), word-non-word cued recall (Mårtensson \& Lövdén, 2011) and face-name cued recall (Mårtensson \& Lövdén, 2011)), as well as working memory (dual-2-back; Jaeggi et al., 2010) and object location memory (Schmiedek et al., 2010). Different tasks measuring spatial abilities were used, assessing mental rotation (Vandenberg \& Kuse, 1978), spatial orientation (Gramann, Müller, Eick, \& Schönebeck, 2005; Guilford \& Zimmerman, 1948) and perspective taking (Hegarty, 2004). Executive functioning was assessed with a task-switching paradigm (King, Colla, Brass, Heuser, \& von Cramon, 2007). These tasks were analyzed by means of two-sample $t$-tests with an alpha level of $\mathrm{p}<0.005$ (corrected for multiple comparisons using Bonferroni correction). 
In addition the Navigation Strategy task measuring route learning performance was used (Wiener, de Condappa, Harris, \& Wolbers, 2013). In that task, participants had to learn a route consisting of four four-way intersections. Each intersection is characterized by two specific landmarks at the diagonal opposite corners of the intersection that support the acquisition of route knowledge. In the training trials, the participants passively observe the correct way through the maze on the screen. In the test trial, participants approach an intersection either from the same direction as during the training phase (same direction trials, SD) or they approach the intersection from another arm (different direction trials, DD). In both conditions they have to indicate how the original route proceeded from there. Six blocks were performed, each of them involved two training and twelve test trials (four SD and eight DD trials). The route stayed the same across all blocks and participants received no feedback about their performance during the task. A detailed description of the task can be found in the reference article. The two conditions can be solved with different navigation strategies. The SD condition can be solved by solely relying on an egocentric strategy, that is, by having associated the direction of turn with the landmarks. The DD condition, in contrast, requires processing the configuration of landmarks and therefore relies on an allocentric navigation strategy. Performance was measured as mean accuracy (percent correct responses) across all blocks in each of the two conditions. The performance difference between SD and DD trials (Navigation Task Difference score) was computed to index the benefit for a given individual in trials that can be solved based on an egocentric strategy compared to trials that afford an allocentric strategy. Performance differences between the groups were analyzed by two sample $t$-tests with an alpha level of 0.05 . To assess the interaction between condition and group we used a mixed ANOVA with condition (ego-/allocentric) as within-subject factor and group as between-subject factor. Statistical outliers were excluded before analysis (outliers were defined as values exceeding the 1 st and 3rd percentile by $>1.5$ times the interquartile range).

The 10-item Edinburgh Postnatal Depression Scale (Cox, Holden, \& Sagovsky, 1987) was used to assess depressive symptoms. It was administered on each measurement occasion to all participants. A 3-item questionnaire assessed the hours the participant slept the day before, the average hours the participant slept during the last two weeks and the average hours the participant slept habitually before pregnancy.

\subsection{MRI data acquisition}

MRIs were acquired using a 3T Magnetom Tim Trio MRI scanner system (Siemens Medical Systems, Erlangen, Germany) using a 12 -channel radiofrequency head coil. High-resolution anatomical images were collected using a T1-weighted 3D MPRAGE sequence $(\mathrm{TR}=2500 \mathrm{~ms}, \mathrm{TE}=4.77 \mathrm{~ms}, \mathrm{TI}=1100 \mathrm{~ms}$, acquisition matrix $=256 \times 256 \times 192$, sagittal $F O V=256 \mathrm{~mm}$, flip angle $=7^{\circ}$, voxel size $=1.0 \times 1.0 \times 1.0 \mathrm{~mm}^{3}$ ).

\subsection{MRI data analysis}

Anatomical data were processed by means of the voxel-based morphometry (VBM) toolbox (http://dbm.neuro.uni-jena.de/vbm. html) with default parameters by Gaser and the SPM8 software package (http://www.fil.ion.ucl.ac.uk/spm). The VBM8 preprocessing involves bias correction, tissue classification, and registration. The 'nonlinear only' modulation was applied in order to preserve the volume of a particular tissue within a voxel by multiplying voxel values in the segmented images by the Jacobian determinants derived from the spatial normalization step. In effect, the analysis of modulated data tests for regional differences in the absolute amount (volume) of gray matter (GM). Finally, images were smoothed with a full-width half-maximum kernel of $8 \mathrm{~mm}$. Statistical analysis was carried out by means of whole-brain comparison of GM volume between the two groups. Age and total intracranial volume were entered as covariates of no interest. The resulting maps were thresholded with $p<0.0001$ and a statistical extent threshold combined with a non-stationary smoothness correction were applied. After computing the whole-brain analysis, the GM values for each participant in the statistically significant regions were extracted by means of MarsBaR region of interest toolbox for SPM (http://marsbar.sourceforge.net/) and correlated with behavioral performance and hormonal levels (Pearson correlations). Whole-brain correlations with estrogen levels during pregnancy as well as following delivery were performed for the peripartal group. These exploratory analyses were performed in order to disentangle effects of pre- vs postpartal estrogen on the brain structure in the peripartal group.

\section{Results}

\subsection{Sample description}

The mean time between the cognitive assessment during the last weeks of pregnancy and day of delivery in the peripartal group was $22.3( \pm 9.3)$ days. The imaging measurement took part on average about $33.5( \pm 8.1)$ days after delivery. The mean distance between the two sessions for the peripartal group therefore was $55.8( \pm 12.7)$ days. Except for three participants, the cognitive and imaging session of participants in the control-group took part within one week. The two groups did not differ with regard to age and educational status $(p>0.1)$ or to their scores on the Edinburgh Postnatal Depression Scale ( $p>0.1$ for both time points). The two groups did not differ in their self-reported hours of sleep in the night and two weeks before the cognitive test-session (during pregnancy for the peripartal group) $(p>0.1)$. At the MRI session, women in the peripartal group compared to the control group reported to have slept significantly less the night before ( mean $=5.5 \mathrm{~h}, t(53)=4.62, p<0.001$ ) as well as the average of the two weeks before $($ mean $=5.8 \mathrm{~h}, t(53)=4.73, p<0.001)$.

\subsection{Hormonal data}

Estrogen was significantly elevated in pregnant women compared to the control group (154.64 $( \pm 61.69) \mathrm{pg} / \mathrm{ml}$ pregnant women; $10.04( \pm 6.56) \mathrm{pg} / \mathrm{ml}$ control group; $t(42)=16.18$, $p<0.001)$. After delivery, women had significantly lower estrogen levels compared to the control group $(2.38( \pm 1.90) \mathrm{pg} / \mathrm{ml}$; $t(41)=5.77, p<0.001)$. Given their non-normal distribution, estrogen scores were log-transformed before analysis.

\subsection{Cognitive tasks}

The groups did not differ reliably in their performance on the memory, spatial and executive functioning tasks $(p>0.005$, see Supplementary Table S1 for details).

Navigation Strategy task data for two participants in the peripartal group were missing due to technical problems. Women in the peripartal group performed significantly less accurate in the SD condition of the Navigation Strategy task (82\% vs $90 \%$ $t(53)=-2.22 ; p=0.031)$. However, the groups did not significantly differ in their DD performance, resulting in a significant group by condition interaction $(F(1,50)=4.93 ; p=0.031$; Fig. 1$)$.

\subsection{MRI data}

A VBM whole-brain comparison between the two groups revealed smaller GM volume in two regions within the left 


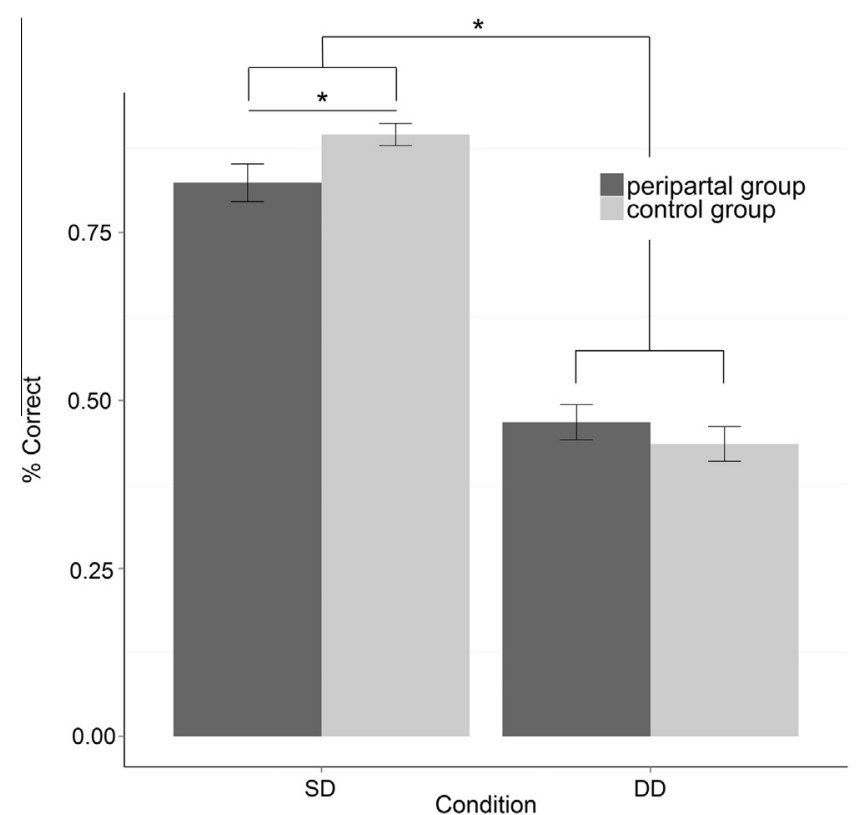

Fig. 1. Performance navigation strategy task. Group by condition interaction for Same Direction (SD, egocentric) Trials compared to Different Direction (DD allocentric) Trials $(p=0.03)$. Error bars represent between-subject standard error (SE). ${ }^{*}=$ significant, $p<0.05$.

striatum (putamen) in the peripartal group compared to the control group $(x=-30, y=-17, \quad z=-2 ; \quad x=-18, \quad y=6, \quad z=-6$; $p<0.0001$, cluster threshold $=$ expected voxels per cluster, $\mathrm{k}>46$, corrected for non-stationary smoothness; Fig. 2). When the threshold was lowered to $p<0.005$, smaller striatal GM was observed bilaterally $(x=20, y=-15, z=-11)$. There were no regions showing larger GM volumes in the peripartal group.

To further explore this finding using anatomically defined regions, GM volumes of the left and right putamen and the left and right hippocampus were extracted. Regions of interest were based on the automated anatomical labelling (AAL) atlas (Tzourio-Mazoyer et al., 2002). Two sample $t$-tests on these data showed that the peripartal group had lower GM volumes of the left and right putamen than the control group $(t(58)=3.08, p=0.003)$, whereas group differences for the left and right hippocampus were not reliable $(p>0.05)$. The GM differences between hippocampus and putamen differed significantly between the groups with greater difference scores (favoring the hippocampus) in the peripartal group $(t(58)=2.72, p=0.009)$. These results should be seen as a complementary analysis to the hypothesis-free whole-brain analysis reported above.

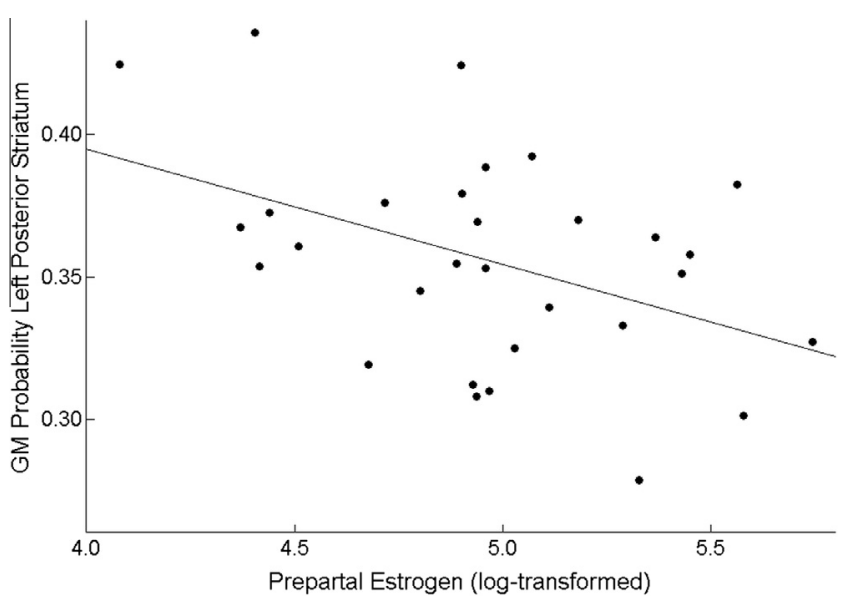

Fig. 3. Striatal gray matter correlation with prepartal estrogen. Gray matter (GM) probability in the left posterior putamen (VBM-ROI) correlated negatively with estrogen measured during late pregnancy, $r=-0.44, p=0.016$

\subsection{Relationship between MRI and hormonal data}

Prepartal estrogen levels in the peripartal group (saliva samples were collected in late pregnancy) correlated negatively with GM probability in the posterior putamen region $(r(28)=-0.44$, $p=0.016$, Fig. 3 ). The GM probabilities in this region did not correlate with postpartal estrogen levels.

To further explore whether GM volume in the striatal region might be associated with pre- or postpartal estrogen, we conducted two whole-brain correlations in VBM with the respective estrogen levels at the time point of MRI acquisition. Three clusters correlated negatively with prepartal estrogen: the left putamen (overlapping with the cluster derived by the group contrast), the right middle temporal gyrus and the right anterior hippocampus $(p<0.001$, cluster threshold $=$ expected voxels per cluster, $k>76$, corrected for non-stationary smoothness). No regions were found to correlate positively with prepartal estrogen and no regions were observed to correlate positively or negatively with postpartal estrogen.

\subsection{Relationship between MRI and cognitive data}

GM probability in the left striatum (the region showing significant differences between the groups in the whole-brain comparison) correlated positively with the Navigation Task Difference score (SD-DD performance) in the total sample $(r(50)=0.30$, $p=0.033$; Fig. 4). Participants with larger striatal GM volume showed a more positive Navigation Task Difference Score, indicat-

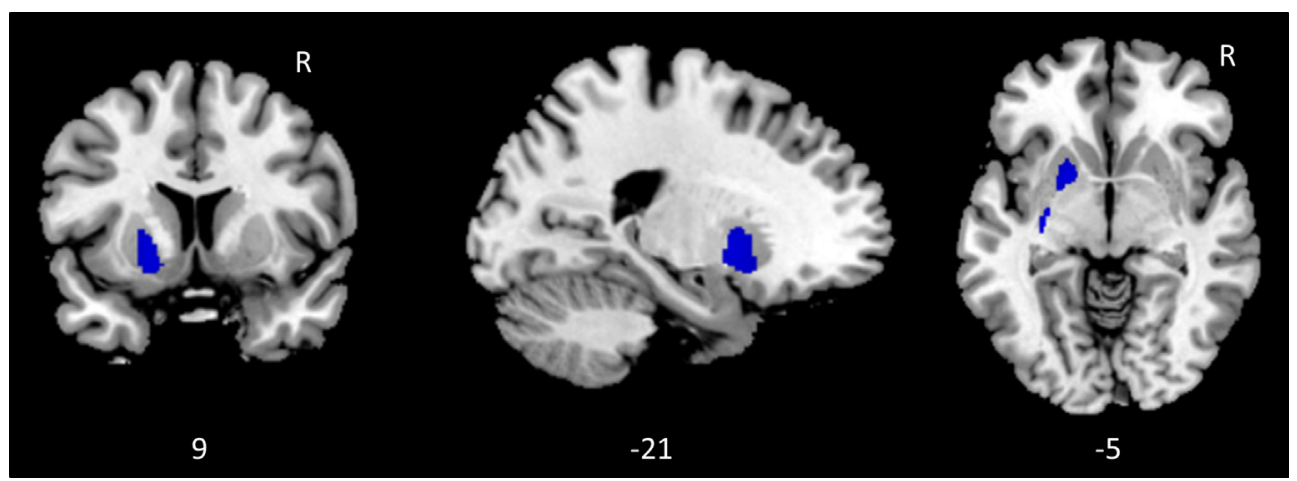

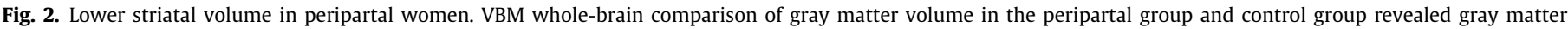
decreases within left striatum in the peripartal group $(p<0.0001$, cluster threshold expected voxels per cluster $(\mathrm{k}>46)$, corrected for non-stationary smoothness). 


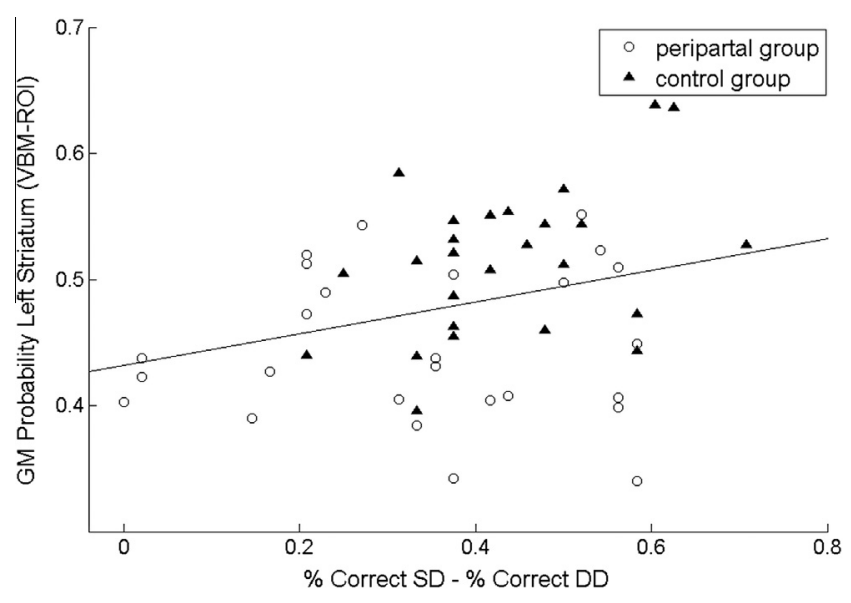

Fig. 4. Striatal gray matter correlation with "egocentric prevalence". Gray matter (GM) probability in the left putamen (VBM-ROI) correlated with the Navigation Task Difference score ("egocentric prevalence" = \% Correct Same Direction (SD) - \% Correct Different Direction (DD)), $r=0.30, p=0.033$.

ing a greater benefit associated with the egocentric task condition (egocentric prevalence). This linear association was not present when assessed within the peripartal group $(r(24)=0.04$, $p=0.850)$, but reliably different from zero in the control group $(r(24)=0.40, p=0.043)$. In addition, the ratio of hippocampal (left and right) over striatal (left and right putamen) GM correlated positively with allocentric task performance $(r(53)=0.3, p=0.029)$. A larger ratio was related to better performance in the allocentric condition. That association was not reliable when analyzed within the peripartal group $(r(25)=0.18, p=0.359)$ and trend-wise significant in the control group $(r(26)=0.35, p=0.070)$.

\section{Discussion}

We investigated navigation performance in pregnant and non-pregnant women in relation to whole-brain structural differences observed between the groups. Pregnant women showed lower performance than non-pregnant women in the egocentric condition of a route learning task, but did not differ in the allocentric condition of the same task. Within the first two months after delivery, left striatal gray matter volume was significantly lower in the postpartal women than in the non-pregnant women. The performance difference between the ego- and allocentric condition correlated positively with gray matter volume in the striatum. Taken together, these results are consistent with the hypothesis that pregnancy goes along with structural changes in navigationrelated neural systems and that these changes influence navigation performance.

As expected, we found that women's route learning performance was altered during pregnancy. This alteration was limited to performance impairments in the egocentric condition of the Navigation Strategy task. Contrary to our expectations, pregnant women did not outperform the control group in the allocentric task condition. We did not observe significant differences in any of the other spatial memory tasks or spatial orientation tasks. The selectivity of the observed group differences should be replicated in future studies, since the long test session and the large number of different cognitive tasks may have hampered the chance to detect small effects due to a reduction in attention especially in the tasks that were performed later during the test session.

We interpret the results from the Navigation strategy task as a shift in strategy use, rather than solely a performance difference in the egocentric condition of the task. The egocentric condition is less difficult than the allocentric condition, as participants approach the way-finding decision points from the same direction as during learning. It is therefore an unusual pattern to find decreased performance in the easier, but not in the more difficult condition. If pregnancy-related performance differences would reflect general problems in memorizing the route, pregnant women would also show lower performance in the allocentric condition of the task. Memory performance was also tested with a variety of other tasks, none of which indicated general decrements in memory performance in pregnant women. Hence, we suggest that the observed pattern of group differences reflects a shift away from egocentric processing in the group of peripartal women. If a participant strongly relies on an allocentric learning strategy, which requires processing the spatial configuration of landmarks at the intersection together with the arm in which the route continues, she might be worse in remembering the simpler stimulus-response association that is sufficient to solve the task in the egocentric condition correctly. Accordingly, the benefit from approaching intersections from the same direction as during learning would be reduced. The performance in the allocentric and egocentric conditions would converge. This pattern was observed in the peripartal group. The direction of this strategy shift is in line with previous results on estrogen's influence on navigation strategies in animals. In rats, estrogen treatment leads to a bias towards hippocampal-based (allocentric) strategies, even when this strategy is disadvantageous in the given context (Daniel \& Lee, 2004; Packard, Hirsh, \& White, 1989).

The hypothesis that pregnancy goes along with a shift in navigation strategy raises the question of a potentially adaptive function of this behavioral change in females. It is difficult to speculate on evolutionary reasons for these observations in humans, because the environment (e.g., during motherhood) has changed a lot in the evolution, but principles of hormonal action on the brain and thus behavior might have persisted. In mammals living in the wild (e.g., rats) the dam has to cope with multiple challenges that require adaptions in her behavior. Foraging and finding the way back to the nest in order to feed the offspring are two examples of behavior necessary for survival of the offspring and therewith for reproductive success. In the functional domain of breeding, changes in the navigation system could likely constitute an evolutionary advantage. In comparison to egocentric navigation strategies, allocentric strategies can lead to the goal even when the well-known route is blocked and thus allow more flexible navigation in a changing environment (Hussain et al., 2014). Allocentric strategies therefore could be beneficial during breeding in animals living in the wild. Clearly this is purely speculative and scientific studies specifically targeted to this question are needed to investigate evolutionary aspects of hormonerelated neural and behavioral changes in females. Comparative behavioral and/or neuroimaging studies may offer a promising approach in this realm.

As hypothesized, we observed differences in neural structure within the navigation network between the two groups. Postpartal women had significantly lower volume in the left putamen, which is located within the striatum. Contrary to expectations, we did not observe significant group differences in hippocampal gray matter volume. Perhaps, our whole-brain measures were not able to uncover fine-grained differences in hippocampal subfields. The observed volumetric differences in the striatum are generally in line with our assumptions of pregnancy-related changes in navigation brain networks. We cannot draw further conclusions about the reasons for these differences, or the neural mechanisms that underlie them. A wide range of animal studies has shown peripartal plasticity of the female brain (Hillerer, Jacobs, Fischer, \& Aigner, 2014). In humans, first evidence confirms brain structural alterations during pregnancy (Oatridge \& Holdcroft, 2002), but whether these changes are exclusively caused by hormonal changes is 
unknown. Longitudinal work is needed to ascertain whether the observed group differences in striatal volume are related to continued exposure of high estrogen levels during pregnancy. The negative correlation of estrogen levels during pregnancy and gray matter volume in the putamen serves as an indicator for this assumption. Hormonal changes occurring during and after delivery (such as the drop in estrogen levels) could potentially also cause the observed brain structural alterations. To elucidate this, estrogen was measured postpartally as well. Only the prepartal estrogen levels correlated (negatively) with striatal gray matter volume. Further research is needed to investigate whether hormonal alterations temporally precede and potentially cause the observed neural changes, and to delineate mechanisms that link the two changes over time. Interestingly, we also observed a negative correlation of prepartal estrogen with the hippocampal head. This part of the hippocampus has not been related to spatial, but rather (verbal) memory and emotional functions (Fanselow \& Dong, 2010; Hackert et al., 2002; Kühn \& Gallinat, 2014). Therefore, this finding could be interesting in light of (mostly verbal) memory deficits during pregnancy that have been observed in some of the previous investigations (Sharp, Brindle, Brown, \& Turner, 1993), although we did not observe significant differences in (verbal) memory performance between the groups in the present study.

Behavioral and brain structural differences were correlated across both groups, as well as within individuals in the control group. Participants with a higher ego-allocentric difference score, indicating a stronger preference for the egocentric strategy, had larger gray matter volumes in the striatal region. Striatal volume was not correlated with either ego- or allocentric performance on its own, but was related to the ratio between the two. In our view this finding suggests that structural changes in the striatum are associated with the tendency to use a specific strategy. We also observed group differences in the proportion of hippocampal over striatal volumes that were positively associated correlated with allocentric task performance. These associations are in line with previous findings in human neuroimaging studies, showing that striatal activity and volume correlates with (egocentric) response learning and hippocampal activity and volume with (allocentric) place learning (Bohbot, Lerch, Thorndycraft, Iaria, \& Zijdenbos, 2007; Hartley, Maguire, Spiers, \& Burgess, 2003; Iaria et al., 2003; Voermans et al., 2004). The absence of a correlation between brain structure and performance when looking at the peripartal group highlight the need for further research and replication. In the present study, the time gap of a few months between the cognitive and the imaging session might have reduced the potential to observe connections within this group.

The present study has several limitations. First, the crosssectional, group-comparative design of the present study data does not permit strong causal inferences about the sequence and direction of associations among changes in hormone levels, spatial learning, and structural brain parameters in the course of pregnancy. The directionality of the links between these three sets of variables is unclear; in addition, third variables may exert a common influence on all three. Pregnant women experience a large number of physiological, psychological and environmental changes that potentially exert influence on brain and behavior that are related to spatial learning and memory. The findings need to be replicated and further inspected by longitudinal studies.

Second, we note that relevant hormonal, structural, and behavioral changes may have taken place during the 55 days elapsing between the prepartal cognitive assessment and the postpartal imaging session (e.g., decline in estrogen levels). These changes may have influenced the present results in ways that are difficult to predict. The MRI measurements after delivery might be influenced (at least to some degree) by the change processes surrounding delivery and thus differ from their state during pregnancy. The time lag between the cognitive and imaging measurement could also have hampered us to find correlations between cognitive and brain structural variables within the peripartal group. For practical reasons, this time lag was unavoidable because ethical considerations prohibited the scheduling of the imaging session during pregnancy. One alternative would have been to shift the cognitive test session closer towards the MRI session, that is, to a time point shortly after birth. However, it is likely that this would have lowered the comparability of the two groups to an even greater extent, as sleep deprivation and distraction due to the likely presence of the baby during testing would have compromised the results. Indeed, the groups differed in reported hours of sleep (during the last night and last two weeks) only at the measurement time point after delivery. Striatal gray matter volume was not associated with the reported amount of sleep, so that we disregard sleep deprivation as a major explanatory factor of the present results. One option to control for the necessary time-gap would be to measure the control-group in a similar time distance between the measurements. This should be considered in future studies.

Third, positive selection bias may have affected the results for the peripartal group. Our sample consisted of women who were able to come to the lab at the very end of pregnancy as well as shortly after delivery. The women in this group may have been positively selected on various dimensions of mental and physical health that are known to relate to the phenomena under study. In fact, this selection bias may help to explain why we do not observe impairments in other cognitive tasks, as observed in previous studies (Henry \& Rendell, 2007). While we would agree that this likely bias weakens the external validity of the present results (e.g., generalization to the population of peripartal women), we contend that it improves their internal validity (i.e., studying the effects of estrogen on brain and behavior).

Taken together, our findings demonstrate differences in striatal volume and navigation performance of pregnant and non-pregnant women that might be related to human pregnancy. We observed a lower prevalence of egocentric strategies in spatial learning in pregnant compared to non-pregnant women. We also found a positive association between striatal volume and egocentric strategy prevalence, which is consistent with animal work on hippocampus-based versus striatum-based differences in spatial navigation strategies. Future longitudinal work is needed to better understand the directionality and time course of hormonal, structural, and behavioral changes in the course of human pregnancy.

\section{Author note}

Nina Lisofsky has been a pre-doctoral fellow of the International Max Planck Research School on the Life Course (LIFE, www.imprslife.mpg.de; participating institutions: MPI for Human Development, Freie Universität Berlin, Humboldt-Universität zu Berlin, University of Michigan, University of Virginia, University of Zurich).

\section{Conflict of interest}

The authors declare no conflict of interest.

\section{Acknowledgments}

We are grateful for the assistance of the MRI team at the MPI Berlin consisting of Sonali Beckmann, Nils Bodammer, Thomas Feg, Sebastian Schröder, Nadine Taube, and we thank our student assistants, Laura Trost and Eva Löblein for their help with data collection and preparation. 


\section{Appendix A. Supplementary material}

Supplementary data associated with this article can be found, in the online version, at http://dx.doi.org/10.1016/j.nlm.2016.08.022.

\section{References}

Bohbot, V. D., Lerch, J., Thorndycraft, B., Iaria, G., \& Zijdenbos, A. P. (2007). Gray matter differences correlate with spontaneous strategies in a human virtual navigation task. The Journal of Neuroscience: The Official Journal of the Society for Neuroscience, 27(38), 10078-10083. http://dx.doi.org/10.1523/JNEUROSCI. 1763-07.2007.

Christensen, H., Leach, L. S., \& Mackinnon, A. (2010). Cognition in pregnancy and motherhood: Prospective cohort study. British Journal of Psychiatry, 196(2), 126-132. http://dx.doi.org/10.1192/bjp.bp.109.068635.

Cox, J. L., Holden, J. M., \& Sagovsky, H. R. (1987). Detection of postnatal depression development of the 10-item Edinburgh postnatal depression scale. The British Journal of Psychiatry, 782-787.

Daniel, J. M., \& Lee, C. D. (2004). Estrogen replacement in ovariectomized rats affects strategy selection in the Morris water maze. Neurobiology of Learning and Memory, 82(2), 142-149. http://dx.doi.org/10.1016/j.nlm.2004.06.001.

Darnaudéry, M., Perez-Martin, M., Del Favero, F., Gomez-Roldan, C., Garcia-Segura, L. M., \& Maccari, S. (2007). Early motherhood in rats is associated with a modification of hippocampal function. Psychoneuroendocrinology, 32(7), 803-812. http://dx.doi.org/10.1016/j.psyneuen.2007.05.012.

DeVoogd, T., \& Nottebohm, F. (1981). Gonadal hormones induce dendritic growth in the adult avian brain. Science, 214(4517), 202-204.

Fader, A. J., Hendricson, A. W., \& Dohanich, G. P. (1998). Estrogen improves performance of reinforced T-maze alternation and prevents the amnestic effects of scopolamine administered systemically or intrahippocampally. Neurobiology of Learning and Memory, 69(3), 225-240. http://dx.doi.org/10.1006/nlme.1998. 3820.

Fanselow, M., \& Dong, H. (2010). Are the dorsal and ventral hippocampus functionally distinct structures? Neuron, 65(1), 1-25. http://dx.doi.org 10.1016/j.neuron.2009.11.031.Are.

Galea, L., \& Kavaliers, M. (1995). Gonadal hormone levels and spatial learning performance in the Morris water maze in male and female meadow voles, Microtus pennsylvanicus. Hormones and Behavior, 29, 106-125.

Galea, L. A., Ormerod, B. K., Sampath, S., Kostaras, X., Wilkie, D. M., \& Phelps, M. T. (2000). Spatial working memory and hippocampal size across pregnancy in rats. Hormones and Behavior, 37(1), 86-95. http://dx.doi.org/10.1006/hbeh.1999. 1560.

Galea, L. A. M., Wide, J. K., Paine, T. A., Holmes, M. M., Ormerod, B. K., \& Floresco, S. B. (2001). High levels of estradiol disrupt conditioned place preference learning, stimulus response learning and reference memory but have limited effects on working memory. Behavioural Brain Research, 126, 115-126.

Gibbs, R. B. (2000). Long-term treatment with estrogen and progesterone enhances acquisition of a spatial memory task by ovariectomized aged rats. Neurobiology of Aging, 21(1), 107-116.

Glynn, L. M. (2010). Giving birth to a new brain: Hormone exposures of pregnancy influence human memory. Psychoneuroendocrinology, 35(8), 1148-1155. http:// dx.doi.org/10.1016/j.psyneuen.2010.01.015.

Gramann, K., Müller, H. J., Eick, E.-M., \& Schönebeck, B. (2005). Evidence of separable spatial representations in a virtual navigation task. Journal of Experimental Psychology: Human Perception and Performance, 31(6), 1199-1223. http://dx.doi. org/10.1037/0096-1523.31.6.1199.

Guilford, J. P., \& Zimmerman, W. S. (1948). The Guilford-Zimmerman aptitude survey. Journal of Applied Psychology, 32(1), 24-34.

Hackert, V. H., den Heijer, T., Oudkerk, M., Koudstaal, P. J., Hofman, A., \& Breteler, M. M. B. (2002). Hippocampal head size associated with verbal memory performance in nondemented elderly. NeuroImage, 17(3), 1365-1372. http:// dx.doi.org/10.1006/nimg.2002.1248.

Hartley, T. Maguire, E. A., Spiers, H. J., \& Burgess, N. (2003). The well-worn route and the path less traveled: Distinct neural bases of route following and wayfinding in humans. Neuron, 37, 877-888.

Hegarty, M. (2004). A dissociation between mental rotation and perspective-taking spatial abilities. Intelligence, 32(2), 175-191.

Henry, J. D., \& Rendell, P. G. (2007). A review of the impact of pregnancy on memory function. Journal of Clinical and Experimental Neuropsychology, 29(8), 793-803.

Hillerer, K. M., Jacobs, V. R., Fischer, T., \& Aigner, L. (2014). The maternal brain: An organ with peripartal plasticity. Neural Plasticity, 2014, 574159. http://dx.doi. org/10.1155/2014/574159.

Hussain, D., Shams, W., \& Brake, W. (2014). Estrogen and memory system bias in females across the lifespan. Translational Neuroscience, 5(1), 35-50.

Iaria, G., Petrides, M., Dagher, A., Pike, B., \& Bohbot, V. D. (2003). Cognitive strategies dependent on the hippocampus and caudate nucleus in human navigation: Variability and change with practice. The Journal of Neuroscience: The Official Journal of the Society for Neuroscience, 23(13), 5945-5952.

Jaeggi, S. M., Studer-Luethi, B., Buschkuehl, M., Su, Y. F., Jonides, J., \& Perrig, W. J. (2010). The relationship between n-back performance and matrix reasoning Implications for training and transfer. Intelligence, 38(6), 625-635.

Keefe, J. O., Burgess, N., Donnett, J. G., Jeffery, K. J., \& Maguire, E. A. (1998). Place cells, navigational accuracy, and the human hippocampus. Philosophical
Transactions of the Royal Society of London. Series B, Biological Sciences, 353, 1333-1340.

Kesner, R., Farnsworth, G., \& DiMattia, B. (1989). Double dissociation of egocentric and allocentric space following medial prefrontal and parietal cortex lesions in the rat. Behavioral Neuroscience, 103(5), 956-961.

King, J. A., Colla, M., Brass, M., Heuser, I., \& von Cramon, D. (2007). Inefficient cognitive control in adult ADHD: Evidence from trial-by-trial Stroop test and cued task switching performance. Behavioral and Brain Functions: BBF, 3, 42 .

Korol, D. L. (2004). Role of estrogen in balancing contributions from multiple memory systems. Neurobiology of Learning and Memory, 82(3), 309-323. http:// dx.doi.org/10.1016/j.nlm.2004.07.006.

Korol, D. L., \& Kolo, L. L. (2002). Estrogen-induced changes in place and response learning in young adult female rats. Behavioral Neuroscience, 116(3), 411-420. http://dx.doi.org/10.1037//0735-7044.116.3.411.

Kühn, S., \& Gallinat, J. (2014). Segregating cognitive functions within hippocampal formation: A quantitative meta-analysis on spatial navigation and episodic memory. Human Brain Mapping, 35(4), 1129-1142. http://dx.doi.org/10.1002/ hbm. 22239.

Küppers, E., \& Beyer, C. (1999). Expression of estrogen receptor- a and b mRNA in the developing and adult mouse striatum. Neuroscience Letters, 276, 95-98.

Lisofsky, N., Mårtensson, J., Eckert, A., Lindenberger, U., Gallinat, J., \& Kühn, S. (2015). Hippocampal volume and functional connectivity changes during the female menstrual cycle. NeuroImage, 118, 154-162. http://dx.doi.org/10.1016/j. neuroimage.2015.06.012.

Logan, D. M., Hill, K. R., Jones, R., Holt-Lunstad, J., \& Larson, M. J. (2014). How do memory and attention change with pregnancy and childbirth? A controlled longitudinal examination of neuropsychological functioning in pregnant and postpartum women. Journal of Clinical and Experimental Neuropsychology, 36(5), 528-539. http://dx.doi.org/10.1080/13803395.2014.912614.

Luine, V. N., \& Frankfurt, M. (2012). Estrogens facilitate memory processing through membrane mediated mechanisms and alterations in spine density. Frontiers in Neuroendocrinology, 33(4), 388-402. http://dx.doi.org/10.1016/j.yfrne.2012. 07.004 .

Mårtensson, J., \& Lövdén, M. (2011). Do intensive studies of a foreign language improve associative memory performance? Frontiers in Psychology, 2(January), 12.

McDonald, R. J., \& White, N. M. (1994). Parallel information processing in the water maze: Evidence for independent memory systems involving dorsal striatum and hippocampus. Behavioral and Neural Biology, 61(3), 260-270. Retrieved from http://www.ncbi.nlm.nih.gov/pubmed/8067981.

McEwen, B. (2002). Estrogen actions throughout the brain. Recent Progress in Hormone Research, 57, 357-384. Retrieved from http://www.ncbi.nlm.nih.gov/ pubmed/12017552.

Oatridge, A., \& Holdcroft, A. (2002). Change in brain size during and after pregnancy: Study in healthy women and women with preeclampsia. American Journal Neuroradiology, January, 19-26. Retrieved from <http://www.ajnr org/content/23/1/19.short>.

Packard, M. G. (2009). Exhumed from thought: Basal ganglia and response learning in the plus-maze. Behavioural Brain Research, 199(1), 24-31. http://dx.doi.org/ 10.1016/j.bbr.2008.12.013.

Packard, M. G., Hirsh, R., \& White, N. M. (1989). Differential effects of fornix and caudate nucleus lesions on two radial maze tasks: Evidence for multiple memory systems. The Journal of Neuroscience, 9(5), 1465-1472.

Pawluski, J. L., Brummelte, S., Barha, C. K., Crozier, T. M., \& Galea, L. A. M. (2009) Effects of steroid hormones on neurogenesis in the hippocampus of the adult female rodent during the estrous cycle, pregnancy, lactation and aging. Frontiers in Neuroendocrinology, 30(3), 343-357. http://dx.doi.org/10.1016/j.yfrne.2009. 03.007.

Protopopescu, X., Butler, T., Pan, H., Root, J., Altemus, M., Polanecsky, M., ... Stern, E. (2008). Hippocampal structural changes across the menstrual cycle. Hippocampus, 18(10), 985-988. http://dx.doi.org/10.1002/hipo.20468.

Restle, F. (1957). Discrimination of cues in mazes: A resolution of the "place-vs.-response" question. Psychological Review, 64(4), 217.

Schmiedek, F., Lövdén, M., \& Lindenberger, U. (2010). Hundred days of cognitive training enhance broad cognitive abilities in adulthood: Findings from the COGITO study. Frontiers in Aging Neuroscience, 2(July), 1-10.

Sharp, K., Brindle, P. M., Brown, M. W., \& Turner, G. M. (1993). Memory loss during pregnancy. British Journal of Obstetrics and Gynaecology, 100 209-215.

Tzourio-Mazoyer, N., Landeau, B., Papathanassiou, D., Crivello, F., Etard, O., Delcroix, N., ... Joliot, M. (2002). Automated anatomical labeling of activations in SPM using a macroscopic anatomical parcellation of the MNI MRI single-subject brain. NeuroImage, 15(1), 273-289. http://dx.doi.org/10.1006/nimg.2001.0978.

Van Hartesveldt, C., \& Joyce, J. (1986). Effects of estrogen on the basal ganglia. Neuroscience E' Biobehavioral Reviews, 10, 1-14.

Vandenberg, S. G., \& Kuse, A. R. (1978). Mental rotations, a group test of threedimensional spatial visualization. Perceptual and Motor Skills, 47(2), 599-604 http://dx.doi.org/10.2466/pms.1978.47.2.599.

Voermans, N. C., Petersson, K. M., Daudey, L., Weber, B., Van Spaendonck, K. P., \& Kremer, H. P. H. (2004). Interaction between the human hippocampus and the caudate nucleus during route recognition. Neuron, 43, 427-435.

Wiener, J. M., de Condappa, O., Harris, M. A., \& Wolbers, T. (2013). Maladaptive bias for extrahippocampal navigation strategies in aging humans. The Journal of Neuroscience: The Official Journal of the Society for Neuroscience, 33(14) 6012-6017. http://dx.doi.org/10.1523/JNEUROSCI.0717-12.2013. 
Wolbers, T., \& Hegarty, M. (2010). What determines our navigational abilities? Trends in Cognitive Sciences, 14(3), 138-146. http://dx.doi.org/10.1016/j. tics.2010.01.001.

Woolley, C. S. (1998). Estrogen-mediated structural and functional synaptic plasticity in the female rat hippocampus. Hormones and Behavior, 34(2), 140-148. http://dx.doi.org/10.1006/hbeh.1998.1466.

Woolley, C. S., \& McEwen, B. S. (1993). Roles of estradiol and progesterone in regulation of hippocampal dendritic spine density during the estrous cycle in the rat. The Journal of Comparative Neurology, 336(2), 293-306. http://dx.doi.org/ $10.1002 /$ cne. 903360210.

Zurkovsky, L., Brown, S. L., Boyd, S. E., Fell, J. A., \& Korol, D. L. (2007). Estrogen modulates learning in female rats by acting directly at distinct memory systems. Neuroscience, 144(1), 26-37. http://dx.doi.org/10.1016/j. neuroscience.2006.09.002 